\title{
PROCEEDINGS OF THE TWENTY-SECOND ANNUAL MEET- ING OF THE AMERICAN SOCIETY FOR CLINICAL INVES- TIGATION HELD IN ATLANTIC CITY, N. J., MAY 5, 1930
}

Liver Fractions in Pernicious Anemia. By RANDOLPH WeST and (by invitation) Marion Howe, New York, N. Y.

It has been shown that the material in liver active in pernicious anemia (activity being measured by reticulocyte response after feeding) is not precipitated by sodium sulphate nor by alkaline silver solutions, is precipitated by phosphotungstic acid, is not precipitated by picric acid and is not removed from the water phase on shaking as picrate against butyl alcohol and ether mixtures.

During the past year we have dissolved the Lilly Liver Extract in water, saturated with solid anhydrous sodium sulphate, filtered and removed sodium sulphate from filtrate by adding alcohol to 60 or 70 per cent by volume. After blowing off alcohol an excess of solid picric acid is added, any precipitate discarded, and the filtrate shaken five times against a mixture of equal parts of ether and butyl alcohol. The water layer after shaking out butyl alcohol with ether and blowing off ether is precipitated in 3 per cent sulphuric acid with phosphotungstic acid and the precipitate regenerated after dissolving in 75 per cent acetone with hot baryta. This material is adjusted to $\mathrm{pH} 5.0$ and evaporated in vacuo to $10 \mathrm{cc}$. (material from 400 grams Lilly Extract). This is poured into $190 \mathrm{cc}$. absolute alcohol. The precipitate has been fed in doses of $500 \mathrm{mgm}$. daily with practically no response. The supernatant fluid is active. On adding $10 \mathrm{cc}$. of hot aqueous baryta to the supernatant fluid an amorphous precipitate forms which is centrifuged down, dissolved in water and largely freed of barium with sulphuric acid. This material is highly active, while the final alcoholic supernatant fluid is almost inert. In one instance reticulocytes rose from 4 to 21 per cent with red cells at 900,000 on $500 \mathrm{mgm}$. daily for four days by mouth.

On hydrolysis with hydrochloric acid a crystalline hydrochloride separates which has the crystal form and empirical formula on combustion of hypoxanthine. Ozazones melting at $156^{\circ}$ and $204^{\circ} \mathrm{C}$. have been obtained.

On feeding a crystalline phosphotungstic regenerated precipitate nearly all activity was lost, though the Molisch reaction and hypoxanthine were present. Similarly there is marked impairment of activity on feeding the precipitate from ammoniacal lead regenerated with $\mathrm{H}_{2} \mathrm{~S}$. Adenosine and the sodium salt of inosinic acid from muscle have been fed without any response. Yeast nucleic acid in 5-gram doses daily has proven inert.

The filtrate after precipitation with lead and ammonia was next used, $680 \mathrm{mgm}$. obtained from 1200 grams of the Lilly extract were injected intravenously, the 
reticulocytes rising from 3 to 40 per cent with red cells rising from 1.4 to 2.3 millions (reticulocyte peak 640,000).

This material is amorphous and probably impure. It is acid to methyl red, is levorotatory (about -15). On combustion C 46.6 per cent, $\mathrm{H} 6.9$ per cent, $\mathrm{N} 10.6$ per cent, $\mathrm{O} 35.8$ per cent; no $\mathrm{S}$ nor $\mathrm{P} ; \mathrm{NH}_{2} \mathrm{~N}+$ before hydrolysis trace, after hydrolysis about 50 per cent of $\mathrm{N}$. Amorphous $\mathrm{Ba}$ and green $\mathrm{Cu}$ salts have been obtained, soluble in water, precipitable from alcohol. The precipitate is heavy with phosphotungstic acid, crystalline, moderate with $\mathrm{HgSO}_{4}$, and alcoholic $\mathrm{PtCl}_{4}$, slight with ammoniacal $\mathrm{AgNO}_{3}$. There is none with picric, falvianic nor picrolonic acids, nor with $\mathrm{AuCl}_{3}$. Amorphous precipitate from alcohol with thallous hydroxide. No reduction $\mathrm{KMnO}_{4}$ in acid, slight in alkaline solution. Diazo and Molisch reactions are negative. On distillation with soda lime vapors given a strong pine splinter reaction. On adding $\beta$ napthol and 2 volumes of concentrated $\mathrm{H}_{2} \mathrm{SO}_{4}$ a strong green fluorescence develops. A careful search of hydrolytic products has failed to reveal thymene, uracil or cytosine; but a substance giving a green copper salt, and precipitable by phosphotungstic acid is still present.

The best fraction is rich in a nitrogenous body with acid properties.

\section{Quantitative Studie of the Number of Cells in the Bone Marrow. By RAPHAEL} IsAACs, Ann Arbor, Mich.

The cells of the fresh bone marrow can be counted with a fair degree of accuracy by dissolving a measured amount of marrow in blood serum and then counting the cells by the usual blood counting technic. Differential counts can be made from films made from the serum suspension. The cells, under these conditions, stain the same as do those of the blood stream and can be identified easily. Data on the cell numbers and types in the normal and various diseases have been correlated. In nephritis there is a reduction in the number of adult red blood cells and polymorphonuclear neutrophilic leucocytes in the bone marrow and an increase in the number of "lymphoid" cells.

Further Observations on the Etiological Relationship of Achylia Gastrica to Perniciou: Anemia. By William B. Castle, and (by invitation) Wilmot C. TownSEND, and Clark W. Heath, Boston, Mass.

Observations have already been reported ${ }^{1}$ demonstrating that by an interaction of normal human fasting gastric contents and beef muscle, both of which are separately ineffective, a substance is produced which will cause a prompt hematopoietic effect and clinical improvement in Addisonian pernicious anemia comparable to that obtained with liver feeding. The nature of this reaction has now been studied by means of observations upon fifteen additional cases of pernicious

${ }^{1}$ Castle, W. B.: Proc. Roy. Soc. Med., 1929, xxii, 58.

Idem: Am. J. Med. Sci., 1929, clxxviii, 748.

Castle, W. B., and Townsend, W. C.: Am. J. Med. Sci., 1929, clxxviii, 764. 
anemia in respect to the nature of $(a)$ the extrinsic factor of the reaction contained in beef muscle and $(b)$ the intrinsic factor present in normal human gastric contents. These observations demonstrate that the proteins precipitated at $\mathrm{pH} 6$ from beef muscle may act as the extrinsic factor. Normal human saliva, and duodenal contents collected by a special technique so as to be free from gastric juice are entirely ineffective when incubated with beef muscle. The essential reaction between gastric juice and beef muscle may take place in neutral solution. Heating the gastric juice to $40^{\circ} \mathrm{C}$. for three days or to from $70^{\circ}$ to $80^{\circ} \mathrm{C}$. for one-half hour abolishes its effectiveness. If normal gastric juice is treated with casein solution ${ }^{2}$ and then with magnesium carbonate, ${ }^{8}$ the pepsin and rennin may be completely removed, leaving behind an unknown factor which, however, can react in neutral solution with beef muscle to produce the hematopoietic substance.

In contrast to our original negative experiments with pig stomach mucosa after autolysis in acid, ${ }^{4}$ it has now been found possible to reproduce the activity of 300 grams of fresh pig stomach mucosa by incubating 25 or 100 grams of the fresh stomach mucosa with $\mathbf{2 0 0}$ grams of beef muscle. These experiments suggest that the effectiveness of pig stomach feeding demonstrated by Sturgis and his associates after our original negative results with autolyzed mucosa, ${ }^{5}$ is the result of postmortem changes within the tissue similar to physiological reactions occurring between normal human gastric juice and beef muscle. The activity of the pig stomach is furthermore apparently not identical with that of liver since heating the former to $100^{\circ} \mathrm{C}$. for five minutes is sufficient to markedly effect its potency in contrast to the lack of effect on liver extract under similar circumstances.

The gastric juice of two patients with blood pictures entirely consistent with the diagnosis of pernicious anemia (sprue and multiple intestinal anastomosis), both of which subsequently showed a typical response to effective liver extracts, has been found in each case to contain normal amounts of hydrochloric acid, pepsin and rennin, but to be entirely incapable of reacting with beef muscle. The irrelevance of any of the existing tests of gastric function for the detection of the essential factor of the normal gastric juice is thus made clear.

The Response Obtained in Healthy Pigeons to the Administration of Substances Effective in Pernicious Anemia. By Janet M. Vaughan and Gulli LindH Muller (by invitation) and George R. Minot, Boston, Mass.

Recent work on the treatment of anemia has facilitated the differentiation of a group of human anemias which must be regarded as due to the deficiency of some

\footnotetext{
${ }^{2}$ Hammarsten, O.: Ztschr. f. physiol. Chem., 1911, lxxiv, 142.

${ }^{3}$ Idem: Ztschr. f. physiol. Chem., 1908, lvi, 18.

${ }^{4}$ Castle, W. B., and Locke, E. A.: J. Clin. Invest., 1928, vi, 2.

${ }^{5}$ Sturgis, C. C., and Isaacs, R.: J. Am. Med. Assoc., 1929, xciii, 747.
} 
substance essential for the normal production of adult red blood cells. Such anemias, including pernicious anemia, are characterized by a megaloblastic hyperplasia of the bone marrow. The bone narrow of the healthy grain-fed pigeon is also of this type. It appeared therefore possible that the administration to pigeons of substances capable of alleviating a deficiency in man associated with megaloblastic hyperplasia of the marrow might provoke a response in the bird similar to that resulting in clinical cases. If such were the case it might provide a biological test for the potency of these substances.

Intravenous administration of a séries of relatively pure liver preparation ${ }^{1}$ known to be effective when given intravenously in pernicious anemia gave a consistent response in fourteen healthy birds. Two pigeons only failed to react to a potent fraction. These two negative results were obtained probably, because it was only possible to give an extremely small dose owing to scarcity of material. The response was characterized by a rapid increase in the circulating reticulocytes, from an average control level of 9.2 per cent to one of about 18 per cent. This was accompanied by a pronounced gain in the birds' weight when less pure preparations were used. Similar effects were obtained when liver extract No. 343 (N.N.R.) was given by mouth, except when sepsis was present, which is in agreement with clinical observations. No such definite responses were obtained when extracts ineffective in pernicious anemia were employed. Negative results were also obtained with intravenous injections of sodium chloride and histamine, preparations of both fractions of Vitamin B (Vitamin $B^{1}(G), B^{2}(F)$, and liver extract No. (N.N.R.)), in which the active principle had been destroyed.

Beef muscle previously digested by healthy gastric juice is as effective as liver in pernicious anemia. Human gastric juice fed pigeons caused no reticulocyte response, but beef muscle promptly did so. Probably the pigeon's gastric juice, like normal human gastric juice, can elaborate from beef muscle material that promotes blood regeneration.

The positive action of those substances effective in pernicious anemia upon the bone marrow of the healthy corn-fed pigeon would appear to be of biological significance.

The Use of Blood Bilirubin Curves as a Test for Liver Function. By George A. HARROP, JR., and (by invitation) E. S. G. BerRon, Baltimore, Md.

The measurement of the rate of disappearance of bilirubin from the blood after intravenous injection has been employed as a method for the determination of liver function. The use of bilirubin, which is a physiological material normally excreted by the liver has obvious advantages over dyes and other foreign substances which have been employed for this purpose. In the past the practical

${ }^{1}$ We are greatly indebted to Dr. Edwin J. Cohn and Dr. Thomas L. McMeekin of the Department of Physical Chemistry of the Harvard Medical School who made the liver preparations and supplied them for our experiments. 
difficulties of dissolving bilirubin so that it might be injected, except in extremely alkaline solution, have prevented its use. A method has now been found for dissolving the material and injecting it at the hydrogen ion concentration of the blood.

When $1 \mathrm{mgm}$. of the material per kilogram of body weight is injected, it is found that the material disappears normally from the blood stream promptly, but that with persons with liver damage this excretion is greatly delayed. Comparison with various other methods for liver function-dye injection, levulose, etc., shows that it is very much more delicate. By this method it has been possible to demonstrate hepatic disturbances particularly in chronic long standing anemia, in mildly decompensated cardiac disease and in acute infections, particularly lobar pneumonia, which were hitherto not suspected.

Electrolyte Studies in Histamine Shock. By DaNA W. Atchley, and (by invitation) Dickinson Richards and E. N. Benedict, New York, N. Y.

Histamine shock was studied in a series of dogs using blood samples drawn before, during and after the effect of the drug. Consistent results were observed as follows:

1. Extraordinary increase in hematocrit with no change in serum protein present; indicating removal of whole serum.

2. Striking increase in undertermined acids (B-A); proved by analysis to be lactic acid.

3. Lactic acid acidosis compensated by $(a)$ increase in total base, $(b)$ decrease in bicarbonate.

The unique response to acidosis by increase of base has been noted by $\mathrm{L}$. J. Henderson and A. Bock in the lactic acid acidosis of prolonged exercise.

The Participation of an Unidentified Tissue Substance in the Formation of Wheals. By H. L. Alexander, St. Louis, Mo.

It has been shown ${ }^{1}$ that if a constant amount of histamine be injected intradermally at different sites, the resulting wheals after a given time will vary greatly in size. The same variation occurs with morphine, ${ }^{2}$ allergens, ${ }^{3}$ etc. In general, larger wheals appear on the trunk than on the extremities. Such wheals are presumably due to the release of a histamine-like substance (H- substance) ${ }^{4}$ by the skin cells at the injected sites. This, in turn, acts on the surrounding capillaries, making them permeable to their contained plasma. This theory, probably correct, does not account for the inconstant size of wheals at different sites. There is no evidence that this is due to differences in skin structure or vascularity. It occurs in dogs as well as in human subjects.

${ }^{1}$ Lewis, T., and Grant, R. R.: Heart, 1924, xi, 209.

${ }^{2}$ Gröbel, A.: Zeit. exp. Med., 1929, lxv, 352.

${ }^{3}$ Alexander, H. L.: Proc. Soc. Exp. Biol. and Med., 1928, xxv, 800.

${ }^{4}$ Lewis, Thos.: The Blood Vessels of the Human Skin and their Responses, London, 1927, Chap. VI. 
On the assumption that some tissue factor other than H-substance contributes to wheal formation, the following experiment was done. Skin removed from an anaesthetized dog was washed, chopped and shaken for two hours in nine parts of 0.9 per cent sodium chloride solution (= skin extract). A histamine phosphate solution 1 to 10,000 also in sodium chloride was prepared (= histamine). Of each of the following $0.02 \mathrm{cc}$. were injected intradermally in a second dog: (a) histamine; $(b)$ skin extract; $(c)$ skin extract + histamine (histamine added to concentration of $(a)$. After fifteen minutes, the increase in size of wheal formation was measured with a planimeter. The average readings of a series were $(a) 0.3 \mathrm{sq} . \mathrm{cm}$., (b) $0.0 \mathrm{sq} . \mathrm{cm} .,(c) 0.8 \mathrm{sq} . \mathrm{cm}$. The increase in $(c)$ was constant in several hundred injections. Atropine and morphine reacted similarly with skin extract. Extract of human skin in fewer experiments likewise enhanced the action of histamine.

It is presumed that there is a substance in the skin, irregularly distributed, that enhances the action of wheal forming substances either directly or through the mediation of H-substance. Chemical analysis shows this to be non-dialyzable. Its identification is not completed.

\section{Changes in the Tissues Following the Administration of Excessive Quantities of} Water. By C. H. Greene and L. G. Rowntree, Rochester, Minn.

Several cases of diabetes insipidus have been observed in which the excessive intake of fluids was accompanied by restlessness, agitation, muscular tremor and the development of epileptiform convulsions. Rowntree has described the experimental production of a similar condition in animals, under the name of water intoxication. We have previously demonstrated that the administration of excessive quantities of water will produce a true dilution of the blood.

The accompanying changes in the tissues were studied in a series of young male white rats of approximately 200 grams body weight. Distilled water, in quantities of 5 per cent of the body weight was administered by mouth or intraperitoneally at half-hourly intervals until convulsions occurred. The rats were then killed by bleeding and determination made of the gross and net weight of the animal, of the weight of the eviscerated carcass comprising the skin, skeleton and musculature and of the weight of the brain, liver, kidney, spleen and remaining viscera. The per cent of total solids in the blood, muscles and other organs was also determined.

In general the changes observed were the same whether the water was given by mouth or intraperitoneally. Comparison of the two experimental series of rats with the normal control series shows that there was an increase in weight of between 25.0 and 27.0 grams on the average, or between 12 and 13 per cent of the total body weight. The changes in the blood were similar to those previously reported.

The increase in the trunk was responsible for the greater portion of the increase in weight. A reduction, in the total solid content in the muscle, on the average from 24.28 to 19.65 and 20.97 per cent respectively suggests that the greater part of the water absorbed was taken up by the muscles. There was a slight increase in 
the weight of the brain with an accompanying decrease in the per cent of solids in that organ. These changes were the most striking. The individual variation in the size of the liver, kidney and spleen was so great as to mask any gross changes in these organs, though there was a slight reduction in the percentage of solids in each case.

Studies in Edema. I. The Mechanism of Water Diuresis in Man. By Frank Fremont-Smith, and (by invitation) MaUrice Fremont-Smith, Mary Elizabeth Dailey, Philip Solomon, DeWitt Stetten, Jr., and Margaret P. CARroll, Boston, Mass.

By parallel determinations of creatinine in serum and in urine, it is possible to calculate the amount of glomerular filtrate formed per hour (Rehberg, P. B., Biochem. J., 1926, xx, 447). This we have done during a standard water diuresis test (200 cc. of water every half hour for three hours), both in normal individuals and in patients with various kinds of edema. In addition we have studied the effects of pituitrin, caffeine and urea. Specific gravity, freezing-point depression, creatinine, urea, and chloride have been determined in serial samples of urine, while freezing-point depression, creatinine, urea, chloride, protein, and total solids have been determined in the blood serum.

The normal response to water drinking is a prompt diuresis exceeding in volume the water intake. Little, if any, dilution of the blood serum occurs. In the presence of edema, at the onset of fever, or after pituitrin injection, however, this normal diuresis does not occur, or is delayed, while a significant dilution occurs in the blood serum.

At the height of water diuresis, when the hourly volume of urine is $700 \mathrm{cc}$. or more, the amount of glomerular filtrate formed may not be appreciably greater than when the urine excreted is only $30 \mathrm{cc}$. per hour.

Vimtrup (Am. J. Anat, 1928, xli, 123) has shown that the average glomerulus in man contains fifty non-anastomosing capillaries, while Richards and Schmidt (Am. J. Physiol., 1924, lxxi, 178) have shown in the frog that variations occur not only in the number of glomeruli functioning at any one time but also in the number of patent capillaries in a given glomerulus. On the basis of the above known anatomy and physiology of the glomerular capillaries, we would emphasize the importance of the distribution of the glomerular capillary bed for the control of tubular reabsorption. Thus it is possible to have the same amount of glomerular filtrate formed from one glomerulus with fifty open capillaries as would be formed in the same time from fifty glomeruli with only one open capillary in each. In the first case, however, all the glomerular filtrate would pass rapidly down one tubule; in the second case the same volume of filtrate would be distributed into fifty tubules each receiving one fiftieth of the total, and the flow down each tubule would be proportionately slower. Cushny (J. Physiol., 1902, xxviii, 431) and others have presented evidence that the slower the rate of flow of glomerular filtrate down the tubule the more will it be concentrated. Hence a small volume of concentrated 
urine would be the result if the filtrate is formed in a large number of glomeruli with but one capillary open in each, while a dilute urine of large volume may be expected if the filtrate is formed in relatively few glomeruli in each of which all or most of the capillaries are open, for example, at the height of water diuresis. Thus a redistribution of a given volume of blood flow from many glomeruli with few capillaries open, to few glomeruli with many capillaries open, could produce the extreme changes in the urine volume and concentration occurring during water diuresis, when only minor changes in the amount of glomerular filtrate occur. A similar redistribution would explain the results of Pickford and Verney (Am. J. Physiol., 1929, xc, 470) who found in a heart-lung preparation that the ligation of a primary arterial branch to the kidney did not produce a reduction in urine volume proportional to the diminution in blood flow. In fact, not infrequently, an absolute increase in urine flow occurred at constant perfusion pressure.

Other conditions being constant, the amount of glomerular filtrate formed in a given time is a linear function of the number of glomerular capillaries patent no matter what their distribution, while the extent of reabsorption in the tubules at any given time is a function of the number of tubules active.

Variations in the distribution of the glomerular capillary bed allows wide range in the number of tubules available for the concentration of any given amount of glomerular filtrate. All the filtrate derived from fifty capillaries may be poured into one tubule or distributed into fifty tubules.

This offers a differential mechanism for the excretion of water and of solids, which, if substantiated, must play an important rôle in the regulation of both the volume and the composition of the organism. The two contrasting clinical conditions, uremia without edema, and edema without nitrogen retention, now become understandable on the basis of functional or structural changes in the distribution of the glomerular capillary bed. On the one hand, the glomerular filtrate is deficient in quantity, but so distributed into relatively few tubules (from glomeruli with many capillaries open in each) that there is little reabsorption. A polyuria of low specific gravity results; edema is absent. The small contracted kidney and the polycystic kidney are examples. On the other hand, as in nephrosis, the glomerular filtrate is normal in quantity, but is always distributed into so many tubules (from glomeruli with but few capillaries open in each) that much reabsorption takes place leading to edema. A small volume of concentrated urine is excreted and water diuresis fails to occur. When both the glomerular filtration surface is deficient and the available glomeruli have but few patent capillaries in each. both nitrogen retention and edema take place.

At autopsy the glomerular pathology is suggestively in accord with this conception (Bell, E. T., Am. J. Path., 1929, v, 587).

The Relation of Edema and of Fatigue to the Potassium Content of Skeletal and Cardiac Muscle. By Tinsley Randolph Harrison and (by invitation) J. Alfred Calmoun, Glenn E. Cullen, and Cobb Pilcher, Nashville, Tenn.

Previous studies have shown that the cardiac and skeletal muscles of individuals 
dying of congestive cardiac failure contain an abnormally small amount of potassium. In the present study an attempt has been made to determine the reasons for this phenomenon.

Edema appears to be the cause of the loss of the potassium from skeletal muscle because:

1. The skeletal muscle of individuals with congestive heart failure has an abnormally high water content.

2. Diminished potassium content of skeletal muscle is found in subjects with edema due to other causes.

3. The potassium content of skeletal muscle of patients who have cardiac disease but who have never had edema is normal.

4. The potassium content of skeletal muscle rises as the patient loses edema.

5. The daily injection of saline solution, or Ringers solution, into one of the hind legs of a dog results after a week or more in a marked decrease in the potassium content of the "edematous" leg-the normal leg showing no such diminution.

These changes are not simply dilution effects as the potassium content of "dry" as well as "wet" muscle is affected.

Edema does not appear to be the cause of the loss of potassium from the cardiac muscle as the water content of the hearts of patients dying of congestive heart failure is usually normal or nearly normal.

The loss of potassium from the cardiac muscle appears to be related to "cardiac fatigue" for the following reasons:

1. In patients with hypertension, but without congestive heart failure the potassium content of the right ventricle has been found to be normal; that of the left ventricle low.

2. In a patient dying of massive collapse of the lung, the potassium content of the left ventricle was normal, whereas, that of the right ventricle was diminished.

3. In a patient with concretio cordis with the peripheral signs of cardiac insufficiency, but with a small atrophic myocardium, the potassium content of both ventricles was higher than normal.

4. Stimulation of the sciatic nerve for several hours results in a diminished potassium content of the muscles of the stimulated leg as compared to the "control" normal leg.

5. Tachycardia resulting from vagotomy may cause diminution in the cardiac potassium.

6. Ligation of one of the primary branches of the pulmonary artery causes a change in the potassium content of the right ventricle but no change in that of the left ventricle.

7. Digitalis causes a change in the potassium content of the heart.

It is believed that the loss of potassium brought about by edema and by overwork is in each instance, related to a state of relative tissue oxygen lack.

Potassium dibasic phosphate was administered to a group of patients with congestive cardiac failure. In those of this group who came to autopsy the skeletal 
muscle contained normal amounts of potassium but the cardiac muscle contained only slightly more than that of a similar group who had not received this salt.

A study of the relationship of potassium loss to other electrolyte changes is in progress.

Studies on the Experimental Transmission of Acute Upper Respiratory Infection.

(Common Cold). By Gerald S. Shibley, A. R. Dochez, and (by invitation)

Katherine C. Minls, New York, N. Y.

Further progress in the experimental transmission of human colds to apes by means of filtrates is presented. In addition, a limited number of transmission experiments in which human subjects were used is reported.

To the present, we have successfully passed colds, by intranasal inoculation with filtered nasal washings, from humans to apes in 44 per cent of the cases attempted. Further, we have transmitted colds from ape to ape by the same method. Attempts to transmit colds have been unsuccessful when we have used filtrates which have been kept from 10 days to 2 weeks and when we have used for purposes of inoculation the Gram-negative filter-passing organisms (described by Olitsky and his co-workers) obtained from patients suffering from colds.

In the human experiments the subjects have been taken singly and have been placed in rigid quarantine. To rule out possible entrance during incubation of colds, and for purposes of preliminary bacteriological study, the subjects have been kept under observation from 5 to 7 days. At the end of this period, filtered nasal washings, obtained from individuals suffering from typical colds, have been inoculated intranasally. To the present, we have been successful in transmitting colds in 43 per cent of the cases attempted.

Variations in the Nasopharyngeal Flora in the Tropics (Virgin Islands, West Indies) during the Period of a Year. By W. G. SMILLIE, Boston, and (by invitation) Frank Milam, New York.

The report is upon part of a study of the influence of environmental factors upon variation in the nasopharyngeal flora. A field research laboratory was established in St. John's, U.S. Virgin Islands, or a small, isolated island in the West Indies. Epidemiological, bacteriological, and climatological observations were continued for one year.

The basic nasopharyngeal flora were found to be similar to the types observed in southern Alabama and Labrador. Some interesting and striking differences were noted. One mild epidemic of colds occurred and was studied. The correlation of the occurrence of upper respiration infections with variations in atmospheric temperature was noted.

The Spread of Rheumatic Fever through Families. By JoHN R. PAUL and (by invitation) Robert Sallinger, New Haven, Conn.

A familial study upon rheumatic fever has been engaged upon in the belief that it might bring us into closer contact with the hereditary and environmental condi- 
tions under which this obscure disease naturally develops, than it is possible to establish through observations upon isolated hospitalized patients.

To attack the problem we studied twelve families. Selection was made on the following bases: (1) that at least two or more members of the family shall have suffered from rheumatic fever, and (2), that all of the members of the family shall have been under observation at the New Haven Hospital or Dispensary for a reasonable period of years.

The method of recording data is that devised by Opie in his studies on the spread of tuberculosis through families.

The character of the distribution of rheumatic fever through these families strongly suggests that we are dealing with a chronic disease which is moderately infectious. The appearance of an active flare-up of rheumatic fever in one member of the family has been frequently accompanied by simultaneous outbursts of the disease in other members and not infrequently by the appearance of non-specific types of illness, such as infectious arthritis, bronchitis, bronchopneumonia unexplained skin rashes, etc.

The spread of the disease occurs more frequently to the younger rather than to the older members of the family.

Serological Reactions in Pneumonia with a Non-protein Somatic Fraction of Pneumococcus. By Winliam S. Tilletr and (by invitation) Thomas Francis, JR., New York, N. Y.

Up to the present time the two chemical constituents of pneumococcus cells employed in immunological and serological reactions have been:

1. Soluble specific substance (Type-specific carbohydrate).

2. Somatic protein (so-called nucleo-protein).

The present report is based upon observations made with a third chemical fraction derived from pneumococci and chemically distinct from the other two (designated Fraction "C'). The chemical nature of Fraction " $C$ " has been determined by Dr. Walther F. Goebel and is reported in a separate communication. At the present time it is sufficient to state that it is non-protein and appears to be a carbohydrate common to the pneumococcus species.

Sera obtained at frequent intervals from patients acutely ill with, or convalescent from pneumonia have been mixed with varying dilutions of Fraction " $C$ " and the presence or absence of precipitation noted. It has been found that serum derived from a patient during the acute phase possesses a high titre of precipitins for Fraction "C." Within a day or two after recovery this precipitating power abruptly and permanently disappears. The sera of 50 patients have been tested at frequent intervals from admission to the hospital until several months after recovery. In every instance the precipitating capacity for Fraction " $\mathrm{C}$ " has run a similar course. The phenomenon is unrelated to the type of pneumococcus causing infection.

The curve of the precipitin titre for Fraction " $C$ " is distinctly different from that obtained by the use of either type-specific carbohydrate or nucleo-protein fractions. 
Type-specific antibodies are absent during the acute phase of pneumonia, are demonstrable at about the time of crisis, and are homologous to the infecting organism. On the other hand, anti-" $C$ " antibodies are highest during the acute stage, disappear just after crisis, and are not related to type specificity. Anti-protein antibodies do not vary markedly during the course of pneumonia. Consequently it is possible to plot three distinct curves of antibody titre depending upon which of three chemically distinct fractions of pneumococcus is used as precipitinogen.

Sera derived from patients suffering from febrile diseases other than pneumonia have also been tested for the capacity to react with Fraction "C." Of the limited number so far investigated, precipitins of Fraction " $C$ " have been found only in those individuals acutely ill with diseases, whose etiological agent is known to be, or suspected of being a Gram-positive coccus. In those instances anti-"C" antibodies are demonstrable during the active febrile states and are not present during periods of normal temperature.

Bacteriological Studies on Rheumatic Fever. By Russell L. CECrn, and (by invitation) Edith E. Nicholls, and Wendeli J. StaInsby, New York, N. Y.

Streptococci have been obtained in a high percentage of patients with rheumatic fever, both from the blood and from the affected joints. Most of these streptococci have been classified as alpha streptococcus (Streptococcus viridans). Occasionally, however, beta or gamma streptococci are isolated. Agglutination and absorption tests indicate that a considerable number of the strains of streptococci recovered from the blood and joints of patients with rheumatic fever show a tendency to fall into definite biological groups. The serum of patients with rheumatic fever usually shows agglutination for the type of streptococcus recovered from the blood and joints. These findings corroborate those of previous investigators and tend to establish the conclusion that rheumatic fever is a streptococcal infection, usually of the alpha or viridans type. The pathogenesis of rheumatic fever in respect to the joint lesions appears to be analogous to that of infectious arthritis and gonococcal arthritis. Bacterial allergy probably influences the clinical picture in all three conditions, but in each instance the joint manifestations are primarily dependent upon localization of bacteria in the joint with subsequent infection.

Experimental Acute Glomerulitis. By FranCIS D. W. LuKens (by invitation) and WARFIELD T. LONGCOPE, Baltimore, Md.

For many reasons it has seemed impossible heretofore to produce with any regularity a diffuse glomerulitis in animals by the intravenous injection of bacterial toxins or bacteria. It was therefore decided to bring these substances, in concentrated form, in more direct contact with the kidney. Filtrates from the growth of haemolytic streptococci, lysates of haemolytic streptococci and dead bodies of haemolytic streptococci were therefore injected directly into the left renal artery in rabbits. The animals were killed at different intervals after the operation. By this method it was possible to produce a unilateral inflammation of intense degree 
which often involved every glomerulus, sometimes affected the interstitial tissues and usually resulted in acute degenerative lesions in the tubular epithelium.

Experimental Studies on Lung Collapse in the Rabbit. By RoBert G. Bloch, (by invitation) and FrankLIN C. MCLEAN, Chicago, Ill.

These experiments were undertaken to study the mechanical intrathoracic effects of lung collapse, the influence on the pleural structure, and the changes in respiration. Seventy normal rabbits were treated over periods of five minutes to eleven months.

The anatomical conditions in the human and the rabbit's chest differ so widely that experimental results can be applied to the human chest only very cautiously.

The average rabbit's lung re-expands almost completely within twenty-four hours after a good collapse with atmospheric air. The periphery expands first. The intervals between filling could gradually be lengthened up to ten days. A marked non-inflammatory thickening of the visceral pleura occurs. It is concluded that the absorption of air is directly proportional to the thickness of the pleura.

The abdominal and thoracic respiration together with the intrapleural pressure were recorded during the procedure of collapse and afterwards. Even extreme collapse does not change the abdominal respiration materially, but increases the thoracic respiration about five times and strains the uncollapsed lung to capacity. These results suggest that treatment by simultaneous bilateral pneumothorax as a clinical procedure should be looked upon with great reserve.

Sympathetic Inhibition of the Large Intestine in Hirschsprung's Disease. By W. J. Merle Scott and J. J. Morton, Rochester, N.Y.

A clinical and experimental study of sympathetic inhibition in the large bowel is presented. In two cases of megalocolon the motor activity of the large bowel was tested by its ability to expel a barium enema. All nervous impulses coming from the lumbar and sacral segments of the spinal cord were then blocked off by novocaine. After the induction of this spinal anesthesia, most of the contents of the large bowel were expelled, showing a marked increase in the motor function. In one of these cases we have subsequently, removed both lumbar sympathetic chains with striking improvement. Before operation this seven year old boy had never had a spontaneous bowel movement. Now his bowels move daily without assistance. It is also shown in cats that the dilated colon, the motor function of which is inhibited by sympathetic impulses (through exposure of the bowel), shows vigorous motor activity upon the induction of spinal anesthesia.

These observations demonstrate that the motor anomaly in at least one type of megalocolon is dependent upon sympathetic inhibition of the large bowel. The effect of lumbar anesthesia on the motor function of the colon provides a test for this type of megalocolon and furnishes a prognosis as to the effect of lumbar sympathectomy in an individual case. 
Total Electrolyte Studies and Hydrogen Ion Concentration in Normal and Abnormal Pregnancy. By JoHn P. Peters and (by invitation) David M. KydD, New Haven, Conn.

The $\mathrm{pH}$ as determined gasometrically on the venous serum of 10 normal pregnant women was found to lie in the same range as the $\mathrm{pH}$ of 8 normal persons. The reduction of bicarbonate content of about 8 volumes per cent was compensated by a fall of about $4 \mathrm{~mm}$. in $\mathrm{CO}_{2}$ tension. In eclampsia the $\mathrm{pH}$ was lowered at the time of the convulsion which is similar to what occurs in severe muscular exercise. Observations of $\mathrm{pH}$ in other toxemias were inconsistent except in cases of simple edema when they were normal.

Total base in the toxemias is not markedly altered from the normal pregnant figure except in convulsions when it is higher than the ordinary level. Total serum proteins are reduced in normal pregnancy entirely at the expense of the albumin fraction, the globulin remaining at about the normal non-pregnant level. Edematous toxemias show serum protein figures below the normal pregnant level, but in the other toxemias there is little change in the serum proteins except in cases of dehydration where, as would be expected, they are elevated.

In cases of vomiting of early pregnancy the serum chloride is not markedly altered and in the vomitus of two cases no free hydrochloric acid and equivalent amounts of base and chloride have been found.

The changes in the acid-base equilibrium appear to be related not to the types of toxemias recognized in the usual classification but to the chief presenting symptoms.

The Dynamics of the Circulation in Patients with Coarctation of the Aorta. By Herrman L. Blumgart, John S. Lawrence, and (by invitation) A. CariTON ERnStene, Boston, Mass.

Although coarctation of the aorta has been diagnosed ante-mortem in almost eighty patients, no studies of the blood flow have hitherto been available. The

TABLE 1

The resting oxygen utilization in subjects with coarctation of the aorta

\begin{tabular}{|c|c|c|c|c|c|c|c|c|c|c|c|c|c|c|c|}
\hline \multirow[b]{2}{*}{ Subject } & \multirow[b]{2}{*}{ Date } & \multicolumn{3}{|c|}{ Brachial artery } & \multicolumn{3}{|c|}{ Antecubital vein } & \multicolumn{3}{|c|}{ Femoral artery } & \multicolumn{3}{|c|}{ Femoral vein } & \multicolumn{2}{|c|}{$\begin{array}{l}\text { Arteriovenous } \\
\text { difference }\end{array}$} \\
\hline & & 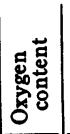 & 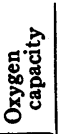 & 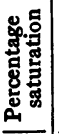 & 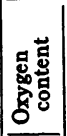 & 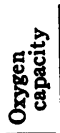 & 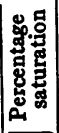 & 荧范 & 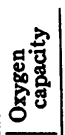 & 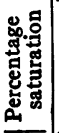 & 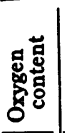 & 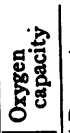 & 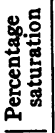 & E & 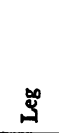 \\
\hline & & $\begin{array}{c}\text { vol- } \\
\text { umes } \\
\text { per } \\
\text { cent }\end{array}$ & $\begin{array}{c}\text { vol- } \\
\text { umes } \\
\text { per } \\
\text { cent }\end{array}$ & & $\begin{array}{c}\text { vol- } \\
\text { umes } \\
\text { per } \\
\text { cent }\end{array}$ & $\begin{array}{c}\text { ool- } \\
\text { wmes } \\
\text { per } \\
\text { cent }\end{array}$ & & $\begin{array}{c}\text { vol- } \\
\text { umes } \\
\text { per } \\
\text { cent }\end{array}$ & $\begin{array}{c}\text { ool- } \\
\text { umes } \\
\text { per } \\
\text { cent }\end{array}$ & & $\begin{array}{c}\text { ool- } \\
\text { umes } \\
\text { per } \\
\text { cent }\end{array}$ & $\begin{array}{c}\text { ool- } \\
\text { umes } \\
\text { per } \\
\text { cent }\end{array}$ & & $\begin{array}{l}\text { vol- } \\
\text { umes } \\
\text { per } \\
\text { cent }\end{array}$ & $\begin{array}{c}\text { vol- } \\
\text { umes } \\
\text { per } \\
\text { cent }\end{array}$ \\
\hline D. W. & $\begin{array}{c}\text { June 26, } \\
1929\end{array}$ & 17.73 & 19.64 & 90.3 & 16.11 & 19.83 & 81.2 & 17.74 & 20.35 & 87.2 & 13.85 & 19.77 & 70.1 & 1.62 & 3.89 \\
\hline J. M. & $\begin{array}{c}\text { Jan. } 7, \\
1930\end{array}$ & 16.07 & 17.86 & 89.9 & 9.71 & 1785 & 54.4 & & & & 5.51 & 17.97 & 31.4 & 6.36 & 10.56 \\
\hline
\end{tabular}


purpose of this communication is to present the clinical findings in two patients with coarctation of the aorta together with the oxygen capacity and content of the arterial and venous blood of the arms as compared to that of the legs, the pulse tracings of the carotid, brachial and femoral arteries, the blood pressure of the brachial, femoral and popliteal arteries, the arteriolar pressure in the upper and lower extremities, and the velocity of blood flow both above and below the coarctation of the aorta as measured by the radium active deposit method.

As in practically all cases of this kind, the arterial pressure above the coarctation was at a hypertensive level whereas the arterial blood pressure in the legs was much lower. The results of the blood gas measurements are shown in the accompanying table (table 1). These results and the measurements of the velocity of blood flow demonstrate that while the blood flow of the legs is diminished it is within normal limits. Our studies indicate that the arterial tension above the site of the coarctation is elevated to a degree sufficient to maintain an adequate blood flow in the areas below the constriction. The elevation of arterial pressure in essential hypertension may well have a similar physiologic significance.

The Order of Excitation of the Ventricles in Bundle Branch Block. By A. G. MacLeod (by invitation), Frank N. Wilson and PaUL S. Barker (by invitation), Ann Arbor, Mich.

In experiments still in progress, it has been found that semi-intrinsic or intrinsicoid deflections may be obtained from the ventricles of the dog when the heart is covered by a pad of gauze, 1.5 to $2 \mathrm{~cm}$. thick, soaked in normal saline solution. Compared with true intrinsic deflections, these deflections are smaller in amplitude and less steep when the recording instrument is used at the same sensitivity. Intrinsicoid deflections can also be obtained from the ventricular cavities by a blood contact. In the case of the auricles, intrinsicoid deflections cannot be obtained either through gauze of the thickness mentioned or from the auricular cavities. It is found that true intrinsic deflections from these chambers are rapidly degraded by increasing the size of the exploring electrode.

The difference between auricles and ventricles is due, so it is suggested, to the difference in the manner in which the excitation process spreads over the ventricular as compared to the auricular muscle.

When right bundle branch block is produced in dogs the intrinsicoid deflections obtained over the right ventricle are late; those obtained over the left ventricle are early. In left branch block the reverse is the case.

In man intrinsicoid deflections can be obtained by placing the exploring electrode upon the precordium. The observations carried out thus far on patients with bundle branch block indicate that in what is at present called right bundle branch block the right ventricle is activated in advance of the left thereby supporting the view 
expressed by Barker, Macleod, Alexander, and Wilson ${ }^{1}$ that the electrocardiograms at present attributed to right branch block are the result of left branch block.

Comparative Sensitivity to Oxygen-Want and to Sodium Lactate of the Hearts of Normal and Thyroxinized Animals. By E. Cowles ANDRus, and (by invitation) Donald McEachern, William a Perlzweig, and Sarah Herman, Baltimore, Md.

The auricles of two rabbits were suspended side by side in a Dale bath. To one animal thyroxin had been administered in graduated doses over a period of two weeks.

The authors have confirmed the observation of J.K. Lewis, that the spontaneous rhythm of the thyroxinized heart persists after isolation at a rate conspicuously more rapid than that of the normal. They have further shown:

1. That the auricles of the thyroxinized animals are far more sensitive to the withdrawal of oxygen than the normal.

2. That their amplitude of contraction is depressed by doses of sodium lactate which produce a little or no effect upon the normal auricles.

3. That recovery from the above takes place more rapidly in the normal than in the thryoxinized auricles.

The authors regard these results as indirect evidence of an accumulation of lactic acid in the heart of the thyroxinized rabbit.

More direct evidence has been obtained by determinations of the lactic acid, glucose and glycogen content of the ventricles of normal and thyroxinized rabbits. The average values are recorded below:

\begin{tabular}{|c|c|c|c|}
\hline & Lactic acid & Glucose & Glycogen \\
\hline & $m g m$. per cenl $l^{*}$ & $m g m$. per cent* & mgm. per cent* \\
\hline Normal (7 experiments). & 34.0 & 164 & 314 \\
\hline Thyroxinized ( 7 experiments)....... & 59.3 & 188 & 116 \\
\hline
\end{tabular}

* Percentage net weight.

These indicate that, in these experiments, thyroxin has brought about an accumulation of lactic acid and a depletion of the glycogen content in the myocardium.

Observations concerning Cerebral Circulation in Man. By WM. G. LenNox and (by invitation) ERNa LeonHardT and AdelaIde BuIst, Boston, Mass.

In more than 100 instances we have measured the gaseous content of blood from the internal jugular vein, and compared it with that of blood drawn from other more superficial vessels. We found that though there was a wide scattering in

${ }^{1}$ Barker, Macleod, Alexander, and Wilson: Trans Assoc. Am. Phys., 1929, xliv, 125. 
individual patients, the average measurements showed that blood leaving the brain was more reduced than blood leaving an extremity.

Calculated respiratory quotients were 0.95 for the brain, 0.82 for the arm and 0.78 for the leg.

When patients breathed pure oxygen, the increase in the oxygen saturation of the blood in the femoral vein was greater than the increase in the internal jugular vein. When a mixture of carbon dioxide and oxygen was breathed the reverse was true. The blood of the femoral vein was not changed, whereas that from the internal jugular became much more like arterial. These correspond with observations by Wolff and Lennox on the response of pial vessels of the cat to similar stimuli.

These observations suggest either a slow blood flow or high metabolic rate of the brain, or both; that the respiratory quotient of the brain is higher than that of skin and muscles; and that the intracranial vessels may react to chemical stimulus in a different maner from vessels in the extremities.

The Circulatory Mechanism and Unilateral Edema in Cerebral Hemiplegia. By Soma WeIss and (by invitation), LAURENCE B. Ellis, Boston, Mass.

Clinical observations frequently reveal vasomotor changes over the paralyzed side in cases of hemiplegia resulting from cerebral vascular lesions. Unilateral edema, usually localized over the upper portion of the body, is often one of the manifestations of alteration in the circulation of such patients with hemiplegia. No extensive study has been made heretofore, however, of the nature and extent of functional alterations in the circulation in the affected part of the body, and no adequate explanation has been offered of the mechanism by which this form of edema is produced.

This communication reports studies of the mechanism of the circulation and the formation of unilateral edema in patients suffering from hemiplegia. The observations indicate that the surface temperature of the body is increased over the paralyzed side of the body, particularly over the upper extremity. The difference between the oxygen content of the cubital artery and vein is always less over the paralyzed side of the body than over the normal side. A similar divergence from the normal in oxygen difference between the femoral arteries and veins is observed to a lesser degree and more infrequently. Microscopic observations of the capillaries of the fingers of the paralyzed side reveal brighter and slightly more prominent skin capillaries, in which the velocity of the blood flow is increased, as compared with the normal side. The arterial pressure of the brachial arteries and the venous pressure in the cubital veins are essentially alike on both sides. The cardiac output in hemiplegia is either normal or slightly below normal. Decrease in the basal metabolism rarely occurs. Circulatory changes may appear immediately after onset of the cerebral accident and last for years. These changes are not necessarily related to the tone of the musculature or to the degree of the paralysis. They occur in the presence both of normal and of elevated blood pressure. The most marked unilateral edema develops in patients with previous myocardial failure. 
It is concluded that in cerebral hemiplegia, following disturbance of the central vasomotor regulation, a dilatation of the arteriolar system of the upper part of the body occurs. The diffuse arteriolar dilatation leads to increased blood flow and increased capillary pressure over the paralyzed side. This increased capillary pressure may lead, especially in association with poor cardiac function, to increased rate of filtration of fluids to the tissue and hence to edema. The functional state of the vessels in the affected side of patients with hemiplegia represents a burden on the circulation which, in the absence of increased cardiac output, ischiefly carried by the normal part of the body. The circulatory changes observed in cases of hemiplegia with marked unilateral edema may have a bearing on the mechanism of the circulation and the formation of edema in congestive heart failure.

A number of nervous, chemical and mechanical responses of the minute vessels of various parts of the body indicate that the vasomotor response and sensitivity of the minute vessels is greater in the upper than in the lower part of the body. This difference in the response of the vessels at various body levels explains the more marked changes in the circulation and the appearance of edema in the upper extremitiy. The fact that the resistance of the minute vessels increases progressively toward the feet is of special teleological and physiological significance in man, whose body is disproportionately high in relation to its size.

The Differentiation and Significance of Certain Ophthalmoscopic Pictures in Bypertensive Diseases. By ArTHur M. FISHBERg, (by invitation) and B. S. OPPENHEIMER, New York, N. Y.

The ophthalmoscopic findings in 280 cases of hypertensive and renal diseases were analyzed in relation to the clinical picture and to the findings at necropsy in 42 of the cases. It was concluded that the traditional unitary conception of "albuminuric retinitis" really includes three pathogenetic entities, the differentiation of which is of diagnostic and therapeutic importance:

1. The arteriosclerotic retinopathy.

2. Malignant hypertensive neuro-retinitis.

3. Choked disk due to edema of the brain.

The ophthalmoscopic pictures of these types of retinal change are described and their occurrence in the individual renal and hypertensive diseases investigated.

In each of the 11 cases of essential hypertension with malignant hypertensive neuro-retinitis that came to necropsy, necrosis of the arterioles of the kidney was present. No instance of essential hypertension with necrosis of the renal arterioles was encountered in which this retinal picture had not been present. However, the same type of retinal change also occurred in glomerulo-nephritis and in a case of suprarenal tumor with hypertension.

The arteriosclerotic retinopathy was found most often in essential hypertension but was also present in chronic glomerulo-nephritis as well as in one case of amyloid contracted kidney.

The process of healing of malignant hypertensive retinitis was followed in one case over a period of four and one-half years. 
Effect of Liberal Carbohydrate Diet upon the Blood Fats in Diabetes. By H. RAwLE GEYELIN, New York, N. Y.

A selected group of fifteen children carefully studied for one to five years prior to the discovery of insulin who have been on a normal diet with insulin ever since, are compared with a group who have been on more of an old fashioned type of diabetic diet and where the blood cholesterol has remained high. We contrast this group with the normal diet group as regards blood fat, cholesterol and sugar and the effect of high carbohydrate upon reducing the blood fat and blood cholesterol in serial determinations after meals and over longer periods of time. The comparative effects of high carbohydrate, low fat diets with the conventional diet upon weight and growth are noted.

The Whole Blood Iron in Normal and Anemic Individuals. By William P. MURPHY, and (by invitation) JoHN POWERS and RALPH LyNCH, Boston, Mass.

In an effort to utilize a more accurate means of following the effect of various types of treatment on the blood of anemic patients other than those with pernicious anemia, a method has been employed for determining the whole blood iron. In addition to the use of this method in following the cases under treatment with various means such as iron in the form of ferrous carbonate, liver, or liver extract and various combinations of these substances, whole blood iron determinations were made on a group of normal individuals in order to attempt to establish a normal whole blood iron figure and a means has been suggested through these determinations of distinguishing between the blood of patients with pernicious anemia and that in patients with so-called secondary anemia.

Observations have also been made which may be made use of in determining the percentage of iron in hemoglobin and so rather accurately the actual hemoglobin content of the blood.

A Study of Inorganic Sulphates in Relation to the Acid-Base Equilibrium in Renal Insufficiency and "Renal Acidosis." By E. G. WAKEFIELD, (by invitation) and N. M. KeITH, Rochester, Minn.

In this work, data have been obtained concerning the concentration of various anions and cations in the blood serum of patients who had renal insufficiency and in a few who had "renal acidosis." The fluctuation of the electrolyte content of the blood serum was less than 15 per cent of the total amount, although wide variations in the concentration of individual ions were observed. In spite of a concentration of inorganic sulphate up to 16 millimolecular equivalents, there was still an undetermined deficit of acid. The fluctuation of the total base was greater than the maximal concentration of inorganic sulphates. There was no constant relationship between concentration of chlorides, phosphates, carbonates and sulphates. Concentrations of lactic acid were low in all the determinations, indicating that the undertermined acid is not lactic acid. 
Creatinine Excretion in Abnormal States of Nutrition. By George Booth, H. B. McCluggage, (by invitation) and Frank A. Evans, Pittsburgh, Pa.

The amount of creatinine excretion in obese patients is independent of their excess weight, and proportional to their ideal weight. During dietary reduction, the creatinine excreted is not changed.

The amount of creatinine excretion in undernourished patients is less than that normal for them if of ideal weight. During dietary correction, the creatinine excretion increases. After attaining a certain level, it remains the same even if the weight continues to increase.

Diphtheria as a Cause of Late Heart Block. By Stuyvesant Butler, (by invitation) and Samuel A. Levine, Boston, Mass.

A study was made of all the cases at the Peter Bent Brigham Hospital and of those seen in consultation by one of us, of complete heart block that were not due to obvious conditions such as coronary thrombosis, fever, rheumatic infection, or digitalis. This limited the group to twenty cases. It was found that in one-half of these cases there was an antecedent history of diphtheria. We have felt for some time that diphtheria was in some way related to subsequent complete heart block, because not infrequently comparatively young individuals have complete heart block, where there is no good cause and where even arterio-sclerosis is absent. Such young patients have given a past history of diphtheria. It is also known that diphtheria in the acute stages can involve the conduction apparatus and produce heart block. Our study lends further support to the view that diphtheria in childhood may be an etiological factor in the development of heart block in later years.

Changes in Cardiac Action During Attacks of Angina Pectoris (Clinical and Experimental Observations). By Francis C. Wood, (by invitation) and Charles C. Wolferth, Philadelphia, Pa.

Twenty-seven,cases of angina pectoris were studied, by means of the electrocardiograph, before, during and after attacks, in an attempt to determine whether there was evidence of some specific change in cardiac action during the attack. Six attacks were spontaneous, 21 were induced by prescribed exertion. As a basis for comparison, the effect of exercise on the electrocardiogram was studied in 160 normals, 25 patients with non-anginal cardiovascular disease, 9 cases of angina in which exercise was not productive of an attack, and 2 patients with non-cardiac pain.

Fourteen of the 27 cases of angina pectoris showed bizarre electrocardiographic changes during the attack, unlike anything seen in the non-anginal cases. Thirteen showed no characteristic alterations during the pain.

The types of electrocardiographic changes, their complete reversibility, and the rapidity of their onset and disappearance were compared with electrocardiograms of dogs with temporary coronary occlusion. A close parallelism was observed.

Anoxemia of a section of a dog's myocardium due to coronary occlusion does 
not always alter the electrocardiogram. Therefore, the absence of significant change in the human electrocardiogram during an attack of angina pectoris cannot be used as evidence that no anoxemia of the myocardium occurred.

The mortality has been overwhelmingly greater in the group with electrocardiographic changes during the attack than in the group without them. This might be considered as added evidence that the bizarre electrocardiograms observed during some anginal attacks are dependent upon an important and significant change in the condition of the heart.

It is concluded, therefore, that the electrocardiograph gives evidence of some unusual change in cardiac action during a certain percentage of anginal attacks, and that the evidence is at least suggestive that this change is due to anoxemia of a section of the myocardium.

The Application of Schütz' Law in Clinical Pepsin Determinations. By J. HARold Austin and (by invitation) GEorge D. GAMMON.

The method of Bloomfield and Polland for pepsin determination has been found useful. The data obtained with either pure pepsin solution or gastric juice conforms approximately to Schütz' law when from one-third to two-thirds of the total edestin has been digested. This fact offers a means of calculating the data into terms of pepsin concentration which is in our hands both more reliable and simpler than the method of calculation suggested by Bloomfield and Polland and appears to us to have a sounder theoretical basis.

The Measurement of Sympathetic Vaso-constrictor Activity in the Lower Extremity. By J. J. Morton and W. J. Merle Scott, Rochester, N. Y.

Vascular diseases in the lower extremity are dependent on two mechanisms; (1) organic occlusion, and (2) functional spasm. For the prognosis and treatment of such conditions it is important to determine the relative participation of these two elements. No entirely satisfactory method for this differentiation is available at present. The skin temperature is used as an index of the circulation to the part.

After spinal anesthesia an increase in the temperature of the foot up to $12^{\circ} \mathrm{C}$. is at times found. The elevation of surface temperature is associated with a flushing of the skin, and is coincidental in time with the appearance of muscular paralysis. It is limited to that part of the body, the motor nerves to which are blocked by novocaine. In purely occlusive vascular disease and in one group of patients without obvious vascular abnormality (particularly cachectic individuals) there is no increase in the surface temperature. We propose the increase of surface temperature in the lower extremity following spinal anesthesia as a measure of the sympathetic vaso-constrictor activity at that point.

A Study of Subcutaneous Nodules in Chronic Infections (Rheumatoid) Arthritis.

By M. H. DAwson, and R. H. Boots, (by invitation) and W. W. Palmer, New York, N. Y.

The occurrence of subcutaneous nodules in patients suffering from rheumatic 
fever has long been recognized and has been the subject of intensive investigation for many years. Carey Coombs has stated that "of the principal phenomena of orthodox rheumatic infection-namely, carditis, polyarthritis, the subcutaneous node, and chorea-the subcutaneous node is the most rheumatic of all." Swift and other investigators have, from time to time, expressed similar views.

Considerably less attention, however, has been paid to subcutaneous nodules occurring in patients suffering from chronic infectious arthritis. During the past year such nodules have been observed in thirty-three patients in the Arthritic Clinic of the Presbyterian Hospital. Nodules have been removed from ten patients and subjected to pathological and bacteriological investigation. All the specimens examined have shown a distinctive and characteristic histological appearance. The various stages in the development of the lesion have been studied and a remarkable resemblance to the nodules of rheumatic fever has been demonstrated. In fact the evidence suggests that the nodules in the two diseases represent different stages in essentially the same pathological process.

The bacteriological investigations have been entirely without result.

The relationship between rheumatic fever and chronic infectious arthritis has also been studied clinically in a group of 300 arthritic patients. The conclusions drawn from these studies also suggest that the two diseases are very closely related and may represent different stages of the same inflammatory process.

\section{Uremic Stomatitis. By PhIlIP S. Hench and (by invitation) Bert E. Hempstead,} Rochester, Minn.

Stomatitis associated with uremia is not described in the English literature, and has been described only three times heretofore, in two French reports and one South American.

Four cases are reported from the Mayo Clinic, with clinical and pathological details. The possible causes of uremic stomatitis and the relation of the oral lesions to uremic ulceration of the intestines and skin are discussed. The various theories regarding uremic stomatitis in general are also discussed.

Uremic stomatitis is a rare condition, but one which it is important for the clinician to recognize as it is of definite diagnostic and prognostic value.

\section{The Clinical Picture of Follicular Lymphoblastoma. By George BAEHR, New York, N. Y.}

A distinctive variety of lymphosarcoma, characterized by the tendency to reproduce lymph follicles of huge size in lymph nodes and spleen is described. The giantfollicles consist of greatly enlarged germinal centers composed of lymphoblasts surrounded by a narrow zone of darker staining mature lymphocytes. They crowd one another so as to compress the intervening sinuses and pulp. The huge spleen, weighing in one case 1800 grams, is thickly studded with follicles the size of barley grains.

The clinical picture is distinguished by the prolonged clinical course and relative benignity, the diffuse involvement of spleen and lymphatic apparatus throughout 
the body, the tendency to thoracic and abdominal effusions, the frequency of unilateral exophthalmos due to involvement of the lachrymal gland or periorbital fat, the absence of cachexia or profound anaemia and the normal blood picture.

The ultimate differential diagnosis is dependent upon microscopic examination of a lymp node removed by biopsy. Histologically, it is sometimes difficult to distinguish from simple hyperplasia. In fact the first three cases were described in 1925, in conjunction with Drs. Brill and Rosenthal, as "Giant Lymph Follicle Hyperplasia of Lymph Nodes and Spleen."

Aside from the distinctive clinical and pathological features, its clinical recognition is important because of its remarkable roentgen sensitivity, the splenomegaly and enlarged lymph nodes promptly melting away under moderate $x$-ray therapy. There is always tendency, however, for the neoplasm to recur in distant parts of the body, though sometimes only after several years.

The chronicity of the disease under treatment is illustrated by three cases followed throughout most of their clinical course. One died nine years after the onset, another after five years, the third is still alive and apparently well after ten years. Death eventually occurs because the lymphatic enlargements become more resistant to treatment or because the neoplasm is located in sites not easily reached by the $x$-ray.

Diabetes Mellitus and Pernicious Anemia. By Howard Root, Brookline, Mass.

The occurrence of 12 cases of combined diabetes and pernicious anemia at the Deaconess Hospital during the last two years led to a search of the literature and finding a total of 32 such cases. A striking feature of the series is that in nearly all cases where accurate histories were available the diabetes preceded the anemia in onset. The clinical features of the two diseases were characteristic, but in certain instances the combination of diseases produced interesting findings. In one case the effect of fasting treatment and the development of diabetic acidosis was to cause a very rapid progress of the anemia and a decrease in the red cells from 3.0 to 1.2 millions in 10 days. An apparent resistance to insulin during the height of the anemia was observed in another case. The rapid development of anemia in a diabetic with a septic leg illustrated the effect of sepsis and diabetes upon a mild and early case of pernicious anemia. A case with advanced symptoms of combined system disease made surprising functional improvement with a combination of dietary, insulin, iron and orthopedic treatment.

In seeking a possible relationship, we determined the hemoglobin of the blood routinely and in patients with diabetes of more than 5 years duration undergoing hospital treatment found a slight reduction in nearly all. The blood smears showed slight variations in the size and shape of red cells and abnormality of the platelets. Cases in this group, however, who had absence of free $\mathrm{HCl}$ in the gastric secretion had reduced red blood counts with much more marked change in the smears. Certain cases of the latter group, under treatment including the use of iron and liver for 2 or more years, have not had relapses or developed symptoms of anemia. The increasing duration of life both of diabetics and of cases of pernicious 
anemia may result in greater frequency of this association merely because the mathematical chances of the association are thus increased, but the earlier onset of the diabetes, and the effect of diabetic treatment upon the anemia suggest that diabetes itself, especially when achlorhydria is present may prepare the way for pernicious anemia.

Some Observations upon the Physiologic and Therapeutic Action of Glycocyamine.

By RalPh H. MajoR, Kansas City, Kans.

Glycocyamine is guanidine acetic acid and differs from creatine only in the absence of the methyl group. Creatine has no effect upon the blood pressure. Glycocyamine has a marked depressor effect.

Observations on capillaries and arterioles show a dilatation after injection of glycocyamine. Observations on the coronary circulation show a very marked increase in coronary flow with a small dose. If the dose is increased to the point of producing a very sudden and intense fall in blood pressure, the coronary circulation is diminished.-The relationship of these observations to the course of treatment in arterial hypertension is commented upon.

A ppearance of Protective Antibodies following Intravenous Injection of Pneumococcus Vaccine in Lobar Pneumonia. By Alvan L. BARAch, New York, N. Y.

The intravenous injection of an antigenic filtrate of the pneumococcus resulted in the appearance of protective substance in the serum of nine patients, seven of whom had lobar pneumonia, on the sixth day and occasionally on the fifth day after injection. After the injection of the intact organism, protective substance appeared on the fourth, and at times on the third day after injection. The immunity increases progressively for three days following injection. This applies to pneumococcus types I, II, and III, and has been produced during the febrile period of the disease.

Clinical Significance of a Large $Q$ Wave in Lead 3. By Harold E. B. Pardee, New York, N. Y.

A large $Q$ wave in the third lead has been observed in records from patients with the anginal syndrome. The frequency of occurrence in such cases is about 27 per cent. Similiar records are obtained from certain patients with rheumatic heart disease, especially those with pericarditis, from a few patients with myocardial fibrosis and congestive heart failure and a few with hypertension without the congestive anginal syndrome. A record with similar large $Q$ waves in lead 3 was found only twice in two hundred and seventy-seven records from apparently normal hearts. In addition to the $Q$ wave of lead 3, these records show a clockwise rotation of the vectors of the QRS group. They usually show a left axis deviation of $Q R S$ and frequently there is an inversion of the $T$ wave in lead 3 or in leads 2 and 3. A discussion of the origin of these features leads to the conclusion that the large Q-3 is due to disease of the muscle of the left ventricle. It is observed, however, that a high position of the diaphragm may be a contributory factor in the 
production of this peculiarity, and that in the normal heart there may be a peculiar distribution of the branches of the auriculoventricular bundle.

The Relationship between the Specific Gravity of the Urine and the Functional State of the Kidneys. By F. H. LASHMET, (by invitation) and L. H. NEwBURGH, Ann Arbor, Mich.

After the subject has been in bed for three days on a standard diet, all food and drink is omitted at 6:00 p.m. of the third day. Thereafter a standard waste in the presence of a limited supply of water is presented to the kidneys for excretion. Normal subjects, eighteen hours later void urine whose specific gravity is 1.025 or more. Nephritic subjects, under the same conditions, have a specific gravity as low as $\mathbf{1 . 0 0 5}$. Correction for temperature is avoided by the use of a pyknometer.

Albumin raises the specific gravity in proportion to its concentration. For example, the specific gravity of the concentrated urine of a nephritic subject was 1.021 before the correction for albumin but only 1.010 after correction. The urine contained 2.5 per cent protein.

If the specific gravity, the blood N.P.N. and phenosulphonephthalein test are compared, it is found that in a group of eighty-six cases with evidence of kidney damage, the blood N.P.N. was normal in 79 per cent; the phthalein test was normal in 30 per cent; but only 10 per cent concentrated the urine specific gravity to 1.025 or above.

We conclude that when the specific gravity of the urine is obtained under the above conditions, it is a much more accurate test of the functional state of the kidneys than the phthalein test or blood N.P.N. and that a roughly quantitative idea of the amount of incapacity may be obtained in this simple way.

Studies on the Colloid Chemistry and Ultramicroscopic Phenomena in Antisepsis and Chemotherapy. By ARTHUR D. HIRSCHFELDER and (by invitation) HAROLD N. WRIGHT, Minneapolis, Minn.

In order to discover, if possible, some of the general principles underlying the phenomena of antisepsis and chemotherapy, we have studied the colloid chemical aspects. We have found that mercurochrome and acriflavine are semi-colloids. The triphenyl-methane dyes are crystalloid, and their fixation by egg albumin, as shown by dialysis experiments, follows the logarithmic curve, which indicates adsorption rather than chemical combination. The fraction absorbed on egg albumin retains some antiseptic action.

The acquired drug fastness toward trypanosomes produced by Browning and Schnitzer with triphenyl-methane dyes against acriflavine, and vice versa, can be duplicated on yeasts in vitro. We believe that this is a surface reaction.

Under the ultramicroscope, particles of lyophillic colloids, like egg albumin, look like soap bubbles; lyophobic colloids look like bright stars and comets. Addition of various antiseptics to egg albumin converts the picture into star and comet appearance like that of a lyophobic colloid. Plasma proteins are better protected but these drugs produce similar, though less marked changes, in vitro and in vivo. Mercurochrome intravenously is fixed by plasma colloids and alters them visibly. 
These studies throw some light on toxic reactions of drugs and other pathological phenomena. We propose to investigate these questions.

Observations on the Intelligence Quotient from the Physician's Viewpoint. By $\mathrm{H}$. Gray, San Francisco, Calif.

Behavior problems and physical diseases in children are less often associated in the physician's mind than they might be. Though no neurological residuals are apparent, there occur at times changes in the brain which the medical man might detect by turning to the intelligence tests in use by psychologists and psychiatrists. In the Institute for Juvenile Research in Chicago, to which are referred children with behavior problems, a search was made for medical diagnoses.

Encephalitis was found in 89 patients with an average IQ of 90.6, which (compared with the average of 90.3 for 2815 cases of all diagnoses) was not significant. If however we consider of this group only those who had mental deterioration, by taking as demarcating line a twelve point drop in IQ, we find 24 cases, and on examining their records for possible medical cause we find three with a history of encephalitis, namely 12 per cent. It is accordingly suggested that a physician who has the care of a child with encephalitis inquire whether an IQ has been determined prior to the illness and if so that he secure a repetition after apparent recovery.

Syphilis was found in 156 children, with an average IQ of 87.8 which (again compared with the average for all diagnoses as stated above) is significantly low.

Delinquency problems are said not to occur in children with IQ's above 130. In this material however 35 such cases are present. Whence it may be urged that children of intelligence must not be considered immune to behavior difficulties.

The physician is sometimes called upon to advise with the school principal as to whether a given child should be pushed up a class or held back. Intelligence quotients are more rapidly obtained, and more standard as between schools, than teachers' marks. Further it is not sufficiently known that children in private schools show distinctly different IQ's from children in public schools, on whom the current standards have been based. In a private school the IQ on 179 children aged 5 to 19 years averaged 114, namely 14 per cent higher than the usual norm of 100.

The Mechanism of the Epigastric Distress Associated with Extragastric Lesion. By Fred M. SMIth, and (by invitation) WM. D. PAUL, and W. M. Fowler, Iowa City, Iowa.

The epigastric distress under consideration is usually localized to the right, and slightly above the umbilicus, and frequently appears from 1 to 3 hours after meals. It is characterized as a feeling of fullness, heaviness, burning, or a gnawing sensation. The site of the distress on fluoroscopic examination, corresponded with the pyloric portion of the stomach and shifts with the change in the position of this region. The association of this distress, with discomfort elsewhere in the abdomen, suggests a reflex stimulation of the stomach. In a former investigation, a reflex 
stimulation of the stomach from gallbladder, appendix and colon was demonstrated in the dog. A consideration of this possibility in man was then investigated. Patients with a spastic colon and a recurring epigastric distress were first selected for study. A balloon was introduced in the pyloric portion of the stomach and connected with a kymograph. After a control record of the gastric activity had been obtained, the colon was distended with air through a rectal tube. There was at once a striking increase in tone and peristaltic action of the stomach, and the appearance of the typical epigastric distress. The pain corresponded with change in tone or the passage of a peristaltic wave over the pyloric portion of the stomach. This induced alteration in the stomach, and the distress promptly subsided after deflation of the colon, or was abolished by atropin. In a patient with chronic appendicitis, similar changes in the stomach, and the typical epigastric distress was induced by massage over the ileocaecal region. This patient was fluoroscoped during the palpation of the appendix region and a striking increase in tone and peristaltic action of the stomach and the appearance of a marked prepyloric spasm was observed and recorded on films. The epigastric distress was experienced during the massage over the appendix, and corresponded with the site of the prepyloric spasm. Similar roentgenological observations were made on patients with an irritable colon. The type of epigastric distress studied is gastric in origin. It is produced by an increase in tone and peristaltic action of the stomach, which is induced by a reflex stimulation from the colon and appendix.

Hemodynamic Studies in a Case of Concretio Cordis. By C. SIDNEy BuRwell and (by invitation) W. D. StrayHORN, JR., Nashville, Tenn.

The patient exhibited signs of obstruction to the flow of blood into the right heart. He had ascites, pleural transudates, peripheral edema, an enlarged liver, distended veins, and a paradoxical pulse. The heart was small, fixed in position and showed no pulsation when observed with the fluoroscope nor any change in electrical axis with shift of position. The sounds were feeble, the pulse small. The lungs exhibited no râles, and the arterial blood was 98 per cent saturated ${ }^{\circ}$ with oxygen. The absence of pulmonary congestion made feasible the measurement of the cardiac output by the acetylene method of Grollman. The venous pressure was measured by the method of Moritz and Tabora.

Under standard "basal" conditions the following observations were made: low pulse pressure $(94 / 82 \mathrm{~mm}$. $\mathrm{Hg}$ ), rapid pulse (104-110), low cardiac output per minute (1.98-2.72 liters), very low output per beat (18-26 cc.), high oxygen utilization (9.0-11.8 volumes per cent, 53-70 per cent utilization), and venous pressure of $240 \mathrm{~mm} . \mathrm{H}_{2} \mathrm{O}$. The venous pressure rose rapidly with the slightest muscular exertion.

When the oxygen consumption per minute was doubled by exercise the following observations were made:

The pulse pressure and output per beat were unchanged, the pulse rate rose to 144, the cardiac output increased only in proportion to the rate, the oxygen utilization rose to 13.0 volumes per cent ( 77 per cent utilization), the venous distension was obviously greater, and the patient was clearly cyanotic. 
After digitalis (1.6 grams) the pulse rate fell to 90 , the cardiac output per minute fell in proportion, the output per beat remained unchanged, and the patient felt less comfortable.

Operation was followed in 12 hours by sudden death. Autopsy revealed a small heart with atrophy of the ventricular myocardium, the whole surrounded by a dense capsule of fibrous tissue which was not adherent to the chest wall but which must have effectually prevented adequate diastolic relaxation.

The symptoms were due to back pressure rather than to diminished volume flow because stasis occurred only in the systemic circulation. The peripheral signs were those of heart failure, the signs in the heart itself were in general the precise opposite of those observed in characteristic congestive heart failure. These observations thus have important bearing on the problem of the mechanism of heart failure.

An Undescribed Cardiac Mechanism: "Intraventricular Block" with Short P-R Interval in Healthy Young People Prone to Paroxysmal Tachycardia. By Louis Wolff, John Parkinson (by invitation) and Paul D. White, Boston, Mass.

Eleven cases are here reported of an unusual cardiac mechanism, heretofore undescribed as such, consisting of functional bundle branch block and abnormally short P-R interval, occurring mostly in otherwise healthy young people with paroxysms of tachycardia or of auricular fibrillation.

Spontaneusly, or following release of vagal tone by exercise or atropinization, the ventricular complexes revert to the normal physiological form, and the P-R interval lengthens (normal).

Vagal influences seem to be largely responsible for the mechanism described. A paradoxical effect of vagal stimulation on the P-R interval has been observed.

From a study of the cases presented it is concluded that:

$a$. Aberrant ventricular complexes of the type generally recognized as indicating bundle branch block may occur in healthy people with normal hearts.

$b$. Vagal stimulation is capable of altering markedly the form of the ventricular complex, and may be responsible for the occurrence of complete bundle branch block curves in apparently normal hearts at normal rates of beating.

c. Vagal stimulation may shorten markedly the P-R interval without the production of A-V nodal rhythm, and without dislocating the auricular pacemaker.

$d$. In the group of cases reported paroxysmal tachycardia and auricular fibrillation are obviously associated with the nervous control of the heart.

Reactions After Administration of Crystalline Insulin. By W. R. CAMPBELL and (by invitation) W. J. Gardiner, and D. A. Scotr, Toronto, Can.

Crystalline insulin was administered in sufficient amounts to patients to induce a lowering of blood sugar. The symptomatology was studied in relation to the level of the blood sugar. As was previously observed, the level at which symptoms appears is about 0.070 per cent. Patients not reaching this level were without 
symptoms. In some instances at this level symptoms were slight and would probably have been overlooked by the patient. No patients reaching hypoglycaemic levels failed to report symptoms and these were more marked with the lower blood sugar levels. In comparison with the crude insulin of 1922 and even the present commercial insulin, reactions are less marked with the crystalline product. No constant effect was noted on the pulse rate or blood pressure; in most cases the values remained unchanged.

Certain patients present initially or develop after a short period of treatment local sensitization phenomena at the site of the injection of insulin. When of minor degree, the patient desensitizes himself. When more severe, it is necessary to change the source of the insulin. One patient, however, shows marked sensitivity to beef, hog, sheep, fish and human insulin obtained from different sources. $\mathrm{He}$ is also sensitive to crystalline insulin though the reaction is less intense. The sensitivity can be locally communicated to other individuals by passive transfer. He has now developed local desensitization on a part of one thigh for crystalline insulin only. Other patients are sensitive to insulin from two or more species and also to crystalline insulin several times recrystallized but the sensitivity in all cases is less marked to crystalline insulin than to other commercial insulins. Inasmuch as the patients are not sensitized to beef muscle, hog muscle, etc., there is probably a sensitization to organ protein as well as to the insulin protein.

Local Immunity in Areas of Skin Hypersensitiveness. By FrankLIN M. HANGER, JR., New York, N. Y.

Rabbits recovering from a skin infection of $B$. lepisepticum are highly immune to re-infection by these organisms. The serum of such animals will protect a local area of skin of a non-immune animal when injected either previously or simultaneously with an emulsion of virulent B. lepisepticum.

We have been interested in studying the effect of local hypersensitive reactions to heterologous proteins on the efficacy of this immune mechanism.

Immune and non-immune rabbits were sensitized to egg albumen by repeated subcutaneous injections. After several weeks, skin sensitivity to egg developed as manifested by the usual raised, red, oedematous areas at the site of intradermal injection of $0.1 \mathrm{cc}$. of egg albumen twenty-four hours previously.

When a thin suspension of $B$. lepisepticum is injected into these areas the infection spreads rapidly throughout the entire wheal in contrast to the much smaller lesion in control normal skin. Even in immune animals with highly protective serum a spread of infection is observed when the local reaction to egg is intense.

When wheals are produced by injecting egg and immune serum simultaneously, infection spreads unchecked. However in non sensitized animals, areas of skin receiving an identical egg-immune serum mixture show complete protection. It is suggested that the serological factors of immunity may be greatly altered by local cellular conditioning. 
The Composition of the Bile Following the Relief of Biliary Obstruction. By CARL H. Greene, and (by invitation) Waltman Walters, and Clyde H. FredRICKSON, Rochester, Minn.

The composition of the bile following the relief of biliary obstruction and its relation to the clinical condition of the patient was studied in a series of nine cases.

The total daily output of bilirubin was more or less constant and was not related to the other factors studied in this series of cases. The concentration, on the other hand, varied inversely with the volume of the bile. One or two patients showed some evidence of a washing out of retained pigment, but if this occurred in all it took place so slowly as not to be apparent in observations of as short duration as these.

Biliary obstruction inhibits or stops the formation of bile acids. If the liver is not too greatly injured there is a relatively rapid return to normal; otherwise the return is greatly delayed. This was true both with regard to the concentration and total amount of bile acids.

The concentration of chlorides in the bile is slightly greater than that in the blood serum. With cholerrhagia, the resultant loss of salts becomes so great as to be of clinical significance. The loss of fluids by this channel may also be so great as to cause a diminution in the output of urine.

The concentration of urea in the bile apparently varies directly with that in the blood. Ordinarily this pathway of elimination is not significant, but in one case with an associated uremia there was a considerable loss of urea through the fistula.

The Heart Rate during Sleep. By ERNST P. BoAs, New York, N. Y.

The heart rates of 51 men and 52 women were studied by means of the cardiotachometer, an instrument that automatically records the heart beats, for periods of 12 to 24 hours each. For men the average minimum heart rate during sleep was 52.7, the average basal rate 61.4. For women the average minimum rate during sleep was 57.7 and the average basal rate 69.9. In 14 of the cases the minimum rate was between 36 and 45 . The degree of reduction in heart rate depends in part on the soundness of sleep as well as upon its duration. Movements during sleep, as well as the impact of various external stimuli, such as noises, determine brief accelerations of heart rate lasting about a minute.

The heart rate is not an absolute measure of the intensity of sleep. The curves of heart rate during sleep have been compared with the curves of depth of sleep reported by other authors. These latter seem to show a rapid increase in the depth of sleep during the first two hours of sleep, followed by a moderate recession, a second peak of deepened intensity in the early morning hours, and then a rapid diminution until awakening. The curves of heart rate show a sharp drop in rate during the first two and one-half hours of sleep, a more gradual drop during the next four hours, followed by a gradual rise during the last hours of sleep before awakening.

The slowing of the heart during sleep is the result of two functions; one a passive one, resulting from the diminution in muscular, mental and emotional activity, in 
reflex excitability, the reduction in temperature and of metabolism; the other an active one, resulting from increased activity of the vagus nucleus, determined, possibly, by the active inhibitory process of the sleep regulating center in the hypothalamus.

Blood Changes in Cases of Severe Burns. By MONROE A. MCIver and (by invitation) RAY F. FARQUHARSON, Rochester, Minn.

The study is based on 20 severely burned cases, showing a mortality of 25 per cent.

Measurements showed that there was no change, early in the illness, in the plasma content of chloride, calcium, phosphorus and non-protein nitrogen. The inorganic constituents of the fluid lost from the burned areas, as shown by the analysis of fluid collected from several large blisters, were found to exist in essentially the same proportions as in normal plasma, and consequently, as might be expected, the loss of the fluid in burns does not alter the chemical composition of the plasma. The protein content was about one-half that of normal plasma.

Since low values of the plasma chlorides have been reported by other authors, a discussion is offered of chloride metabolism in patients with burns.

Marked concentration of the blood was noted, as estimated by the haematocrit readings, and a slowing of the rate of sedimentation of the red cells.

It has been urged by Underhill and others that the picture of acute intoxication following severe burns is due not to the absorption of a toxin but to the great concentration of the blood. While not denying the importance of this latter factor and the need for combating it, our studies seem to show that since in many cases bringing the concentration of the blood back to normal does not prove a lifesaving measure, there are other very important factors in the production of the clinical picture.

The Life Bistory of a Diabetic. Who Acquired An Unusual Tolerance to Insulin. By EDward H. Mason, Montreal, Can.

A case report including autopsy findings is presented of a diabetic male who acquired an extreme tolerance to insulin. The diabetic history was of five years' duration and followed infection of the biliary tract. Evidence of decreasing pancreatic function progressed rapidly, necessitating the use of 200 to 400 units daily of insulin during the last eight months of life. With recurrent attacks of acidosis the insulin requirement increased to a maximum of 2075 units in 24 hours. Death resulted from delayed hypoglycaemia.

Autopsy showed a very small fibrotic pancreas containing advanced calcareous degeneration. A cyst $2 \times 1.5 \mathrm{~cm}$. was found in the ventral part of the mid-brain which suggested a possible relationship between the sympathetic tracts involved and the extreme tolerance to insulin. 
Fluorescence of the Skin as a Protective Mechanism in Pellagra. By Roy H. TURNER, New Orleans, La.

Sunshine is generally admitted to be the most important precipitating cause of the dermatitis of pellagra. The ultraviolet portion of the spectrum has recently been shown to be the most injurious. The observations to be reported were undertaken in an effort to explain instances of apparent immunity in pellagrins to injury by sunshine. The face, though the most exposed to light, is rarely injured and then only mildly. Skins with thick, horny layers seem protected. Castle has shown that keratin and sebum are fluorescent. This quality makes them protective against ultraviolet light, since these rays are changed to visible rays in passing through fluorescent substances.

A series of normal persons and pellagrins have been examined in a dark room by ultraviolet light. Normally the most fluorescent areas are the palms, soles and face, which are areas remarkably free from the dermatitis of pellagra. Early skin lesions of pellagra were non-fluorescent, while the later lesions with roughened skins were highly fluorescent. Lesions at this stage have been shown very resistant to light. Experiments to show that sebum and keratin are the fluorescent substances will be reported.

The Effect of Ergotamine on the Response to Adrenalin in Normal Human Subjects under Basal Conditions. By JoHn B. Youmans and (by invitation) W. H. TrImble, Nashville, Tenn.

Our previous studies have shown that under basal conditions ergotamine, in doses of $0.5 \mathrm{mgm}$. subcutaneously, has no significant effect on the metabolic rate or blood sugar level of normal human subjects but causes a small drop in pulse rate and a slight rise in blood pressure. In the present study we have found that under similar conditions ergotamine tends to diminish or delay the increase in metabolic rate and blood sugar which results from the injection 0.5 to $1 \mathrm{mgm}$. of adrenalin. Ergotamine had little or no effect upon the increase in heart rate caused by adrenalin but augmented slightly the blood pressure raising effect of the latter.

The Response of Blood Platelets to the Administraton of Viosterol. By L. D. Tномpson (by invitation) and D. P. BARR, St. Louis, Mo.

An increase in the number of platelets following exposure of experimental animals to sunlight or ultraviolet light has been noted by a number of investigators (Cramer and Drew, Laurens, Sooy and Sanford). Other observers have noted changes in coagulation time following the administration of cod liver oil (Brougher) and viosterol (Selye) but have made no note of increase of platelets. Phillips and Robertson of Washington University reported an increase in platelet count in the rat following administration of viosterol. Our study was undertaken to determine the effect of viosterol upon the platelet count of man under normal conditions and the presence of certain diseases.

A single oral dose of viosterol causes a slight rise in platelet count in twelve to twenty-four hours which is followed by a fall to approximately normal and a 
secondary rise reaching a maximum on the fourth to fifth day. The maximum count varies from 600,000 to 900,000 . The decline is sharp and the number of platelets reaches normal limits by the sixth to eighth day. The response to single doses of 7,15 and 22 minims is essentially the same. A similar response can be obtained a second time in a given individual after an interval of fourteen days. The response to daily doses of 10 minims differs from that following the single dose. Both the rise and decline in the number of platelets are more gradual but the maximum is reached at about the same time, namely five to seven days. On the ninth to eleventh day the count is within normal limits and continues so for at least three weeks except for irregular, transient increases of 100,000 to 200,000 . The bleeding time and coagulation time are decreased coincident with the increase in platelet count.

In splenectomized individuals, the spleen having been removed 18 months to 6 years previously, the response to viosterol is exaggerated. After the administration of a single dose, the rise in platelets is sharp and continuous and reaches a maximum of $1,400,000$ to $1,600,000$. The decline is also more rapid than normal.

In hemophilia, an approximately normal response to daily doses has been seen. Even in the presence of 800,000 platelets, however, there has been no change in symptoms, bleeding has continued and the red blood cell count has readily fallen.

In thrombocytopenic purpura, the platelet count, the coagulation time and clot retraction may be held within normal limits by viosterol but showers of purpuric spots and hemorrhages from mucous membranes have continued.

Studies have been made and are being continued on a variety of blood dyscrasias. The presence of jaundice does not prevent the normal platelet response to viosterol. In aplastic anemia, the platelets may rise above normal at a time when the number of red and white blood cells is rapidly falling.

The Effect of Sodium Salicylate on Intradermal Reactions of Rabbits. By O. E. Hagebush, (by invitation) and R. A. Kinsella, St. Louis, Mo.

Sodium salicylate is commonly used in the treatment of infections, especially those presumed to be due to invasion by streptococcus. The old idea that acute rheumatic fever is due to infection by streptococcus, and the recently developed conception that the disease is involved in a process of allergy to streptococcus, stimulate a study of sodium salicylate in relation to allergy to streptococcus.

In this study rabbits were inoculated with cultures of a strain of Streptococcus hemolyticus of low virulence. The injections were made into one of the knee joints, $0.1 \mathrm{cc}$. of broth culture being used.

For intradermal tests, $0.1 \mathrm{cc}$. of filtrate from a 5-day culture in Harley's medium was used. Areas near the spine were used for inoculation.

All animals were tested for native reactivity to the filtrate before being employed in the experiments. Fresh animals giving positive dermal reactions were discarded.

In the use of sodium salicylate, 0.2 gram per kilo in 5 per cent aqueous solution was given intravenously. The injections were made more slowly and given daily. 
Preliminary studies showed that following the intra-articular injection of streptococci, purulent arthritis invariably resulted and persisted until the death of the animal. Blood cultures were rarely positive during the life of the animal unless an intercurrent disease such as "snuffles" intervened. When this occurred, hemolytic streptococci were found in the blood at autopsy. Ten days after arthritis was established, positive intradermal reactions were always present.

The conditions of the experiment were severe due to the nature of the infection and the death rate was high in all series. This did not seem to be considerably influenced by the use of sodium salicylate.

In the first series of animals 15 controls gave strongly positive intradermal reactions 10 days after the production of arthritis, and 8 animals, given sodium salicylate 24 hours before the production of arthritis and at 24 hour intervals thereafter, showed slight or no intradermal response.

At this point the results of studies made by Griffin of the reciprocally antagonistic action of benzoic acid and glycin on the growth of white rats, were considered. According to this author the deleterious effect of benzoic acid on the growth of young white rats was removed by adding glycin to the diet. The amount of glycin used was 0.47 gram per kilo, in 4 per cent aqueous solution.

Groups of rabbits were therefore selected, of which one was composed of controls, one of animals receiving sodium salicylate intravenously, one of animals receiving glycin intravenously and one of animals receiving sodium salicylate and glycin freshly mixed in proper solutions. In this experiment, 16 control animals gave strongly positive reactions; 28 animals receiving sodium salicylate, gave slight or no reactions; 4 animals receiving glycin alone gave strongly positive reactions; and 16 animals receiving mixtures of glycin and sodium salicylate, gave strongly positive reactions.

- This effective suppression of so-called allergic reactions by sodium salicylate depends on factors as yet unknown. There is no reason to ascribe this effect to a general depressive action inasmuch as rabbits did not lose weight or appear less healthy while receiving sodium salicylate over a period of 10 days than did controls.

Since the dermal reaction is not heightened by the use of glycin alone, the dermal responses obtained in animals treated with sodium salicylate and glycin combined, must be present because glycin neutralizes sodium salicylate in vivo.

The nature of the dermal reaction itself remains obscure. Whatever the mechanism by which sodium salicylate suppresses the derma! reaction, this mechanism does not effect the nature of the vascular pathology found in these animals. The late proliferative vascular lesions were the same in the various series.

When sodium salicylate was discontinued the dermal reactions tended to return, but after 20 days in 7 animals, only a slightly positive reaction was present, while after 50 days only a moderate reaction was present in 4 surviving animals. When sodium salicylate was administered to 4 animals which had well developed dermal reactions the effect was a gradual suppression of the dermal reaction. 
The experiments thus indicate that salicylates would be more effective in preventing the development of an allergic state following a primary infection than in removing allergy after the primary infection has fully developed.

If acute rheumatic fever is a disease in which the process of allergy is involved then derivatives of salicylic acid might conceivably be beneficial by interfering with this process of allergy. That this interference is of too little importance to modify the vascular pathology is apparent. By implication the overlying process of allergy is unimportant and the underlying focus of infection, at present reputed to be of streptococcal origin, is very important. In turn, the results of cultures of blood and joints become of great importance-a state of the question now many years old.

From these considerations it seems possible to draw the following conclusions:

1. Sodium salicylate suppresses the allergic dermal reactions of rabbits to filtrates of hemolytic streptococcus. This effect is most definite when sodium salicylate is given before the focus of infection has developed.

2. There is no relation between the presence or absence of this dermal reactivity and the character of the vascular pathology. 